\title{
Pre-pregnancy BMI, gestational weight gain and breast-feeding: a cohort study in China
}

\author{
Xing-Yong Tao ${ }^{1,2}$, Kun Huang ${ }^{1,2}$, Shuang-Qin Yan ${ }^{3}$, A-Zhu Zuo ${ }^{1}$, Rui-Wen Tao ${ }^{1}$, Hui Cao ${ }^{3}$, \\ Chun-Li Gu ${ }^{3}$ and Fang-Biao Tao ${ }^{1,2, *}$ \\ 'Department of Maternal and Child Health, School of Public Health, Anhui Medical University, 81 Meishan Road, \\ Hefei 230032, People's Republic of China: ${ }^{2}$ Anhui Provincial Key Laboratory of Population Health \& Aristogenics, \\ Hefei, People's Republic of China: ${ }^{3}$ Department of Maternal and Child Health Centres, Ma'anshan Maternity and \\ Child Health Care Hospital, Ma'anshan, People's Republic of China
}

Submitted 22 June 2016: Final revision received 14 0ctober 2016: Accepted 18 October 2016: First published online 7 December 2016

\begin{abstract}
Objective: The purpose of the present study was to examine the influence of maternal pre-pregnancy BMI and gestational weight gain (GWG) on initiation and duration of infant breast-feeding in a prospective birth cohort study.

Design: Breast-feeding information was collected at 1, 3, 6 and 12 months postpartum. The association of pre-pregnancy BMI and GWG with delayed lactogenesis II and termination of exclusive breast-feeding was assessed with logistic regression analysis. The risk of early termination of any breast-feeding during the first year postpartum was assessed with Cox proportional hazards models.

Setting: Urban city in China.

Subjects: Women with infants from the Ma'anshan Birth Cohort Study ( $n$ 3196).

Results: The median duration of any breast-feeding in this cohort was 7.0 months. Pre-pregnancy obese women had higher risks of delayed lactogenesis II (risk ratio $=1.89 ; 95 \%$ CI 1.04, 3.43) and early termination of any breast-feeding (hazard ratio $=1.38 ; 95 \%$ CI $1.09,1.75$ ) adjusted for potential maternal and infant confounders, when compared with normal-weight women. No differences in breast-feeding initiation or duration of exclusive breast-feeding according to pre-pregnancy BMI were found. Moreover, GWG was not associated with any poor breast-feeding outcomes.

Conclusions: The present study indicated that pre-pregnancy obesity increases the risks of delayed lactogenesis II and early termination of any breast-feeding in Chinese women.
\end{abstract}

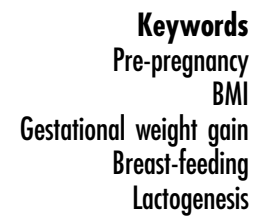

Although the benefits of breast-feeding for both infants and mothers have been extensively documented, the breastfeeding rates in many countries still fall short of the WHO recommendations ${ }^{(1,2)}$. In China, although the breast-feeding rate started to increase in the 1990s, the national target of exclusive breast-feeding (EBF) of $80 \%$ was not reached in most cities and provinces ${ }^{(3)}$. In central and western China, less than one-third of infants younger than 6 months have been exclusively breast-fed ${ }^{(4)}$. The low breast-feeding rates may be partly explained by the increase in obesity among pregnant women. In China, the prevalence of overweight/ obesity is low (approximately $2 \cdot 1 \%$ ), according to the criteria of the $\mathrm{WHO}^{(5)}$. However, the prevalence of obesity increased from $5 \cdot 0$ to $10 \cdot 1 \%$ in women during 1993-2009, according to the WHO suggestions for Chinese ${ }^{(6)}$.

Numerous studies on the association of pre-pregnancy BMI with breast-feeding have mainly focused on the populations of the USA, European countries and Australia $^{(7)}$. Most reports have supported a negative association between pre-pregnancy obesity and the duration of $\mathrm{EBF}^{(8,9)}$ and any breast-feeding $(\mathrm{ABF})^{(8-11)}$. Previous studies have also revealed ethnic differences in breast-feeding success among American women of different pre-pregnancy BMI categories ${ }^{(12-14)}$. Additional studies are required for a comprehensive understanding of the association between pre-pregnancy BMI and breastfeeding among different races/ethnicities ${ }^{(15)}$. Moreover, little information is available on the current situation of breast-feeding in Asian women with pre-pregnancy obesity, including Chinese women.

Compared with pre-pregnancy BMI, which represents the level of fatness before conception, gestational weight gain (GWG) is an indicator of the fat gained during pregnancy. The role of GWG in breast-feeding initiation 
and duration remains unclear. Some studies have reported that excessive GWG exerts a negative effect on breastfeeding practice ${ }^{(16,17)}$. However, other studies have failed to support this effect ${ }^{(9,18,19)}$. The inconsistent findings may be partly because of the number and type of confounders that are controlled for in statistical analyses. Additional studies are required to discern the unique and independent contribution of GWG to breast-feeding outcomes.

Moreover, the timing of the onset of copious milk secretion, known as lactogenesis II, is crucial for successful breast-feeding ${ }^{(20)}$. Delayed lactogenesis II is usually defined as the onset of copious milk secretion $72 \mathrm{~h}$ postpartum. Previous studies have found an association between maternal postpartum obesity and delayed lactogenesis $\mathrm{II}^{(21,22)}$. Two studies with small sample sizes have also indicated that high pre-pregnancy BMI or having a 'heavy body build' is associated with delayed lactogenesis II ${ }^{(23,24)}$. In women with gestational diabetes mellitus, maternal pre-pregnancy obesity has been shown to be a key risk factor for delayed lactogenesis $\mathrm{II}^{(25)}$. Few reports have focused on the relationship between GWG and delayed lactogenesis II.

Thus, the purpose of the current prospective longitudinal cohort study was to examine the relationship among maternal pre-pregnancy BMI, onset time of lactogenesis II, and initiation and duration of breast-feeding in Chinese women after adjustment for known maternal and infant factors. The study also examined whether GWG is independently associated with breast-feeding.

\section{Materials and methods}

\section{Study population}

The study population for the present analysis was drawn from participants of the Ma'anshan Birth Cohort Study in China. The objective of the study was to investigate the effects of prenatal exposure on adverse pregnancy outcomes, child health and development. At recruitment, participants comprised women attending prenatal care clinics affiliated with Ma'anshan Maternity and Child Health Hospital in Ma'anshan City. Women eligible for inclusion into the study were those who initiated prenatal care prior to 16 weeks of gestation. Women were ineligible if they were younger than 18 years of age, did not plan to carry the pregnancy to term, or did not plan to deliver at the research hospital. From 9 October 2013 to 10 September 2014, 3474 eligible women were approached. As shown in the subject flow diagram (Fig. 1), 162 women with pregnancy terminations or stillbirths were excluded. Of the 3312 women who had a live birth, 116 were ineligible due to multiple pregnancies, maternal mortality, missing pre-pregnancy BMI, no delivery and neonatal records, missing breast-feeding data or giving up breast-feeding due to maternal disease. The final sample of postpartum women participating in the study was 3196.

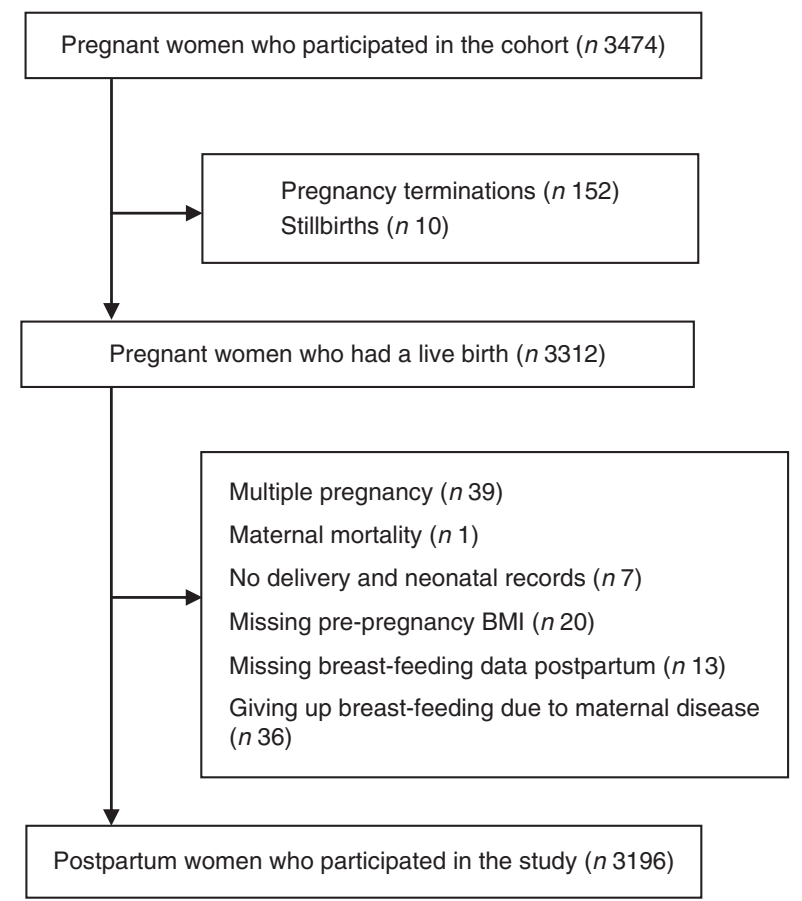

Fig. 1 Flow diagram showing the selection of subjects from the Ma'anshan Birth Cohort Study

At 1, 3, 6 and 12 months postpartum, 99.6, 97·8, 96.6 and $95.3 \%$ of breast-feeding data were available, respectively. The study was approved by the Biomedical Ethics Committee of Anhui Medical University and informed consent for participation was obtained from the mother at the commencement of the study.

\section{Pre-pregnancy BMI and gestational weight gain variables}

Maternal pre-pregnancy weight was self-reported at the first prenatal visit and maternal height was measured. Prepregnancy BMI was calculated as $\mathrm{kg} / \mathrm{m}^{2}$ and categorized according to the Chinese classification as underweight $\left(<18.5 \mathrm{~kg} / \mathrm{m}^{2}\right)$, normal weight $\left(18.5-23.9 \mathrm{~kg} / \mathrm{m}^{2}\right)$, overweight $\left(24.0-27.9 \mathrm{~kg} / \mathrm{m}^{2}\right)$ and obese $\left(\geq 28.0 \mathrm{~kg} / \mathrm{m}^{2}\right)$. GWG was calculated as the difference between pre-pregnancy weight and the last register of weight at delivery. GWG category was also according to Chinese-recommended weight gain ${ }^{(26)}$. Underweight women category should aim to gain $15 \cdot 0-22.0 \mathrm{~kg}$ during pregnancy; those with normal weight, $13.0-21.0 \mathrm{~kg}$; the overweight, $10 \cdot 0-18.0 \mathrm{~kg}$; and the obese, $9 \cdot 5-17 \cdot 0 \mathrm{~kg}$. Maternal GWG categories were then defined as excessive, sufficient and insufficient weight gain.

\section{Breast-feeding variables}

The non-initiation of breast-feeding was defined as no breast-feeding postpartum at all and was assessed at 1 month postpartum. Maternal perception of the onset 
of lactation was also evaluated. Mothers were asked to indicate on what day postpartum they first felt that their breasts were noticeably full after delivery. The timing reported was categorized as three variables ( $<24 \mathrm{~h}, 1-3 \mathrm{~d}$, $>3 \mathrm{~d}$ ). It is a valid method of determining timing of lactogenesis II and has been shown to correlate with actual timing of this stage ${ }^{(27,28)}$. Delayed lactogenesis II is defined as lactogenesis II onset more than $3 \mathrm{~d}$ postpartum $^{(21,24)}$.

At the 1-, 3-, 6- and 12-month follow-ups, the frequency of $\mathrm{EBF}$ and $\mathrm{ABF}$ was evaluated by a questionnaire survey with the guardian. EBF included infants fed breast milk and allows the infant to receive oral rehydration salts, drops and syrups (vitamins, minerals, medicines), but nothing else, as described by $\mathrm{WHO}^{(29)}$. ABF was defined as breast-feeding in combination with infant formula, other milk and/or solid food.

\section{Maternal and infant characteristics}

A self-administered questionnaire was used to collect information on sociodemographic factors and reproductive history in the first trimester of pregnancy. Covariates were selected for their potential influence in the relationship between obesity and breast-feeding, including maternal age, ethnicity, education, parity, household income, smoking status and alcohol consumption. After delivery, the mothers' and infants' charts were systematically reviewed to collect data on pregnancy and delivery, including mode of delivery, pregnancy-induced hypertension and gestational diabetes status, infant sex, gestational age and birth weight. Diagnoses of pregnancyinduced hypertension or gestational diabetes were made by specialized physicians.

\section{Statistical analysis}

The characteristics of the cohort were summarized with unadjusted percentages for categorical data. Pearson's $\chi^{2}$ test was used to test differences between the three categorized BMI groups regarding characteristics of the study population. A stacked bar chart was utilized for the three ranges of onset time of lactogenesis II in women initiating breast-feeding based on different pre-pregnancy BMI groups. Unadjusted and adjusted risk ratios (RR) for onset of lactogenesis II and sustained EBF at 1, 3 and 6 months postpartum according to pre-pregnancy BMI category were calculated through the use of logistic regression models.

Kaplan-Meier curves and log-rank tests were used to evaluate the relationships between pre-pregnancy BMI and GWG groups and duration of breast-feeding. Then, the hazard ratios (HR) between BMI and breast-feeding outcomes after controlling for potential confounding variables were calculated by Cox proportional hazards models. We adjusted the logistic regression and Cox proportional hazards models for the following categorical variables: maternal age $(<25,25-35$ or $\geq 35$ years old), gestational age $(<37$ or $\geq 37$ weeks), birth weight $(<2500$ or $\geq 2500 \mathrm{~g}$ ), mode of delivery (vaginal or caesarean), infant sex (male or female), GWG (inadequate, adequate or excessive), ethnicity (Han or other), education (less than high school, high school, junior college or more than junior college), monthly income of family $(<2500$, 2500-3999 or $\geq 4000$ RMB), parity (primiparous or multiparous), cigarette smoking (never or former/current), alcohol drinking (yes or no), pregnancy-induced hypertension (yes or no) and gestational diabetes (yes or no). All statistical analyses were performed using the statistical software package SPSS version 13.0. Statistical significance was defined as two-sided $P<0.05$.

\section{Results}

\section{Characteristics}

Among the 3196 pregnant women included in our analysis, 605 (18.9\%) were classified as underweight, 300 (9.4\%) as overweight and eighty-two (2.6\%) as obese. The mean age of the pregnant women was 26.4 (SD 3.6) years. The mean gestational age and birth weight of infants were 39.0 (SD 1.4) weeks and 3367 (SD 443) g, respectively. Maternal and infant characteristics according to BMI category are reported in Table 1 . In higher BMI groups, socio-economic status, based on household income and maternal education, was lower. In contrast, in higher BMI groups, maternal age and the rate of caesarean delivery, multiparity, pregnancy-induced hypertension and gestational diabetes were higher. Compared with normalweight women, the overweight or obese women were more likely to have an excessive GWG, premature or low-birth-weight infant.

\section{Breast-feeding initiation and onset time of lactogenesis $\boldsymbol{I}$}

Overall, the rate of breast-feeding initiation was $95.9 \%$. No statistical difference was observed in the rates of breast-feeding initiation among women with prepregnancy underweight (95.0\%), normal weight (96.4\%), overweight (94.7\%) and obesity (93.9\%; $P=0.22)$. Similarly, no statistical difference was found in the rates of breast-feeding initiation among women with insufficient (94.8\%), sufficient (95.7\%) and excessive (96.9\%; $P=0 \cdot 13)$ weight gain. In women initiating breast-feeding, the proportions of onset time of lactogenesis II were $33.0 \%$ for $<24$ h postpartum, $58.3 \%$ for $1-3 \mathrm{~d}$ postpartum and $8.7 \%$ for $>3 \mathrm{~d}$ postpartum. In higher pre-pregnancy BMI groups, the onset time of lactogenesis II was longer (Fig. 2). After adjusting for potential maternal and infant confounders, the RR of delayed lactogenesis II was 0.84 (95\% CI $0.58,1.22$ ) for underweight, 1.38 (95\% CI 0.90 , $2 \cdot 12$ ) for overweight and 1.89 (95\% CI 1.04, 3.43) for 
Table 1 Characteristics of the included participants from the Ma'anshan Birth Cohort Study ( $n$ 3196) by pre-pregnancy BMI category

\begin{tabular}{|c|c|c|c|c|c|}
\hline & \multicolumn{4}{|c|}{ Maternal pre-pregnancy BMI category } & \multirow[b]{2}{*}{$P$ value } \\
\hline & Underweight (\%) & Normal weight (\%) & Overweight (\%) & Obese (\%) & \\
\hline Total $n$ & 605 & 2209 & 300 & 82 & \\
\hline Maternal age (years) & & & & & $<0.001$ \\
\hline$<25$ & $41 \cdot 2$ & 31.6 & $26 \cdot 7$ & $20 \cdot 7$ & \\
\hline $25-35$ & 57.5 & 64.5 & 67.0 & $72 \cdot 0$ & \\
\hline$\geq 35$ & 1.3 & 3.9 & $6 \cdot 3$ & $7 \cdot 3$ & \\
\hline Ethnicity & & & & & 0.856 \\
\hline Han & 98.5 & 98.5 & 98.0 & 97.6 & \\
\hline Other & 1.5 & 1.5 & $2 \cdot 0$ & $2 \cdot 4$ & \\
\hline Education & & & & & 0.014 \\
\hline Less than high school & 18.5 & $19 \cdot 4$ & $26 \cdot 7$ & $26 \cdot 8$ & \\
\hline High school & $25 \cdot 3$ & 21.5 & $25 \cdot 0$ & $22 \cdot 0$ & \\
\hline Junior college & $30 \cdot 1$ & $31 \cdot 8$ & $25 \cdot 7$ & $31 \cdot 7$ & \\
\hline More than junior college & $26 \cdot 1$ & $27 \cdot 3$ & $22 \cdot 7$ & 19.5 & \\
\hline Monthly household income (RMB) & & & & & 0.044 \\
\hline$<2500$ & $24 \cdot 3$ & $26 \cdot 1$ & $26 \cdot 7$ & 41.5 & \\
\hline 2500-3999 & $42 \cdot 3$ & 43.7 & 43.3 & $36 \cdot 6$ & \\
\hline$\geq 4000$ & 33.4 & $30 \cdot 2$ & $30 \cdot 0$ & $22 \cdot 0$ & \\
\hline Infant sex & & & & & 0.484 \\
\hline Male & $51 \cdot 2$ & $51 \cdot 2$ & $47 \cdot 0$ & 54.9 & \\
\hline Female & $48 \cdot 8$ & $48 \cdot 8$ & 53.0 & $45 \cdot 1$ & \\
\hline Gestational age (weeks) & & & & & $<0.001$ \\
\hline$<37$ & $3 \cdot 1$ & $3 \cdot 3$ & $7 \cdot 7$ & 11.0 & \\
\hline$\geq 37$ & $96 \cdot 9$ & $96 \cdot 7$ & $92 \cdot 3$ & 89.0 & \\
\hline Birth weight $(\mathrm{g})$ & & & & & 0.022 \\
\hline$<2500$ & 3.0 & 1.8 & $3 \cdot 0$ & $6 \cdot 1$ & \\
\hline$\geq 2500$ & $97 \cdot 0$ & 98.2 & $97 \cdot 0$ & 93.9 & \\
\hline Mode of delivery & & & & & $<0.001$ \\
\hline Vaginal & $59 \cdot 0$ & 49.8 & $38 \cdot 4$ & 19.5 & \\
\hline Caesarean & 41.0 & $50 \cdot 2$ & $61 \cdot 7$ & 80.5 & \\
\hline Parity & & & & & $<0.001$ \\
\hline Primiparous & $95 \cdot 0$ & $90 \cdot 0$ & $84 \cdot 0$ & $76 \cdot 8$ & \\
\hline Multiparous & $5 \cdot 0$ & $10 \cdot 0$ & $16 \cdot 0$ & $23 \cdot 2$ & \\
\hline GWG* $^{*}$ & & & & & $<0.001$ \\
\hline Inadequate & $23 \cdot 6$ & $16 \cdot 0$ & $9 \cdot 3$ & $15 \cdot 9$ & \\
\hline Adequate & $53 \cdot 7$ & $58 \cdot 3$ & $51 \cdot 7$ & $51 \cdot 2$ & \\
\hline Excessive & $22 \cdot 6$ & $25 \cdot 7$ & 39.0 & $32 \cdot 9$ & \\
\hline Cigarette smoking & & & & & 0.366 \\
\hline Never & $94 \cdot 7$ & $96 \cdot 0$ & $96 \cdot 7$ & 93.9 & \\
\hline Former/current & $5 \cdot 3$ & 4.0 & $3 \cdot 3$ & $6 \cdot 1$ & \\
\hline Alcohol drinking & & & & & 0.762 \\
\hline Yes & $91 \cdot 1$ & $92 \cdot 3$ & 91.7 & $92 \cdot 7$ & \\
\hline No & $8 \cdot 9$ & $7 \cdot 7$ & $8 \cdot 3$ & $7 \cdot 3$ & \\
\hline Pregnancy-induced hypertension & & & & & $<0.001$ \\
\hline Yes & $2 \cdot 1$ & 5.4 & 11.3 & $29 \cdot 3$ & \\
\hline No & 97.9 & 94.6 & 88.7 & $70 \cdot 7$ & \\
\hline Gestational diabetes & & & & & $<0.001$ \\
\hline Yes & $6 \cdot 0$ & $12 \cdot 9$ & $22 \cdot 7$ & 35.4 & \\
\hline No & $94 \cdot 0$ & $87 \cdot 1$ & $77 \cdot 3$ & $64 \cdot 6$ & \\
\hline
\end{tabular}

GWG, gestational weight gain.

${ }^{*}$ Categorized according to guidelines from Chinese-recommended weight gain: inadequate $G W G$ = weight gain of $<15 \cdot 0,<13 \cdot 0$, $<10.0$ and $<9.5 \mathrm{~kg}$ for underweight, normal-weight, overweight and obese women, respectively; excessive $\mathrm{GWG}=$ weight gain of $>22.0,>21.0,>18.0$ and $>17.0 \mathrm{~kg}$ for underweight, normal-weight, overweight and obese women, respectively; adequate GWG = weight gain between the cut-off values for inadequate and excessive GWG.

obese, when compared with the normal-weight group. However, the rate of delayed lactogenesis II was not significantly different among insufficient (7.5\%), sufficient (7.4\%) and excessive $(8.5 \% ; P=0.47)$ weight gain groups.

\section{Breast-feeding duration}

At 1,3 and 6 months postpartum, the rates of EBF were $43.8,51.6$ and $11.0 \%$, respectively. Among the different
BMI groups, obese women had a higher rate of EBF than did normal-weight women only at 1 month postpartum $(\mathrm{RR}=1 \cdot 62,95 \% \mathrm{CI} 1 \cdot 01,2 \cdot 59)$. Moreover, the difference became not significant after adjusting for antenatal characteristics (Table 2). Kaplan-Meier analysis also showed that no significant difference existed in the rate of EBF among the different BMI groups $(P=0.739$ for logrank test). Overall, the median duration of $\mathrm{ABF}$ was 7.0 months. Figure 3 shows the Kaplan-Meier curves for 
the percentage of $\mathrm{ABF}$ across the 12 months of observation when the groups are stratified by BMI. The median duration of $\mathrm{ABF}$ for pre-pregnancy underweight, normal weight, overweight and obesity was 7.0, 7.0, 6.0 and 3.0 months, respectively. A significant difference was observed among the four BMI groups by log-rank test $(P<0 \cdot 01)$. The multivariable Cox proportional hazards regressions showed that the $\mathrm{HR}$ of weaning were 0.99 (95\% CI $0.90,1.10)$ for underweight, 1.11 (95\% CI 0.95 , 1.29) for overweight and 1.38 (95\% CI 1.09, 1.75) for obese, when compared with the normal-weight group. We also examined whether GWG was associated with the continuation of breast-feeding. However, in all analyses, GWG was not a significant predictor for early termination of $\mathrm{EBF}$ or $\mathrm{ABF}$, and did not interact with

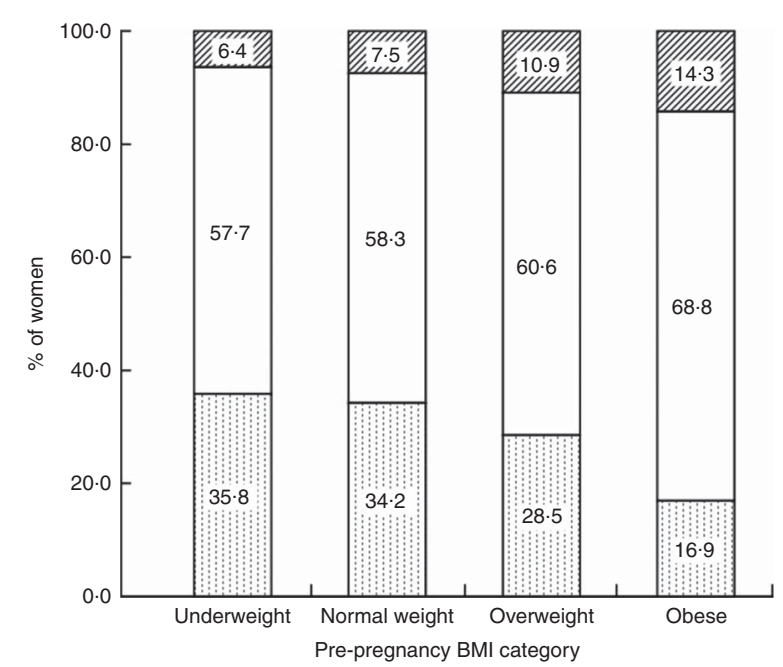

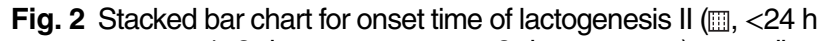
postpartum; $\square, 1-3$ d postpartum; $\square,>3$ d postpartum) according to pre-pregnancy BMl category in women initiating breast-feeding ( $n$ 3065) from the Ma'anshan Birth Cohort Study pre-pregnancy BMI to affect the duration of breast-feeding (data not shown).

\section{Discussion}

The present longitudinal study found that compared with normal-weight women, pre-pregnancy obese women had higher risks of delayed lactogenesis II and early termination of ABF. However, GWG was not associated with poor breast-feeding outcomes. The results mainly support previous findings on the association between pre-pregnancy $\mathrm{BMI}$ and the duration of $\mathrm{ABF}^{(8-11)}$.

The rate of delayed lactogenesis II in the present study $(8.7 \%)$ is close to that in our previous report (9.8\%) in another city of China ${ }^{(30)}$, but much lower than that reported for American women (23-44\%) in studies with small sample sizes ${ }^{(21,22,24)}$. Our study, which included a large sample from the general population, is one of the few studies to examine the effect of pre-pregnancy BMI on lactogenesis $\mathrm{II}^{(23,25)}$. Many studies have primarily focused on the relationship between pre-pregnancy BMI and breastfeeding initiation, and most of them have indicated that pre-pregnancy obesity is associated with decreased breastfeeding initiation ${ }^{(8,16,19,31-33)}$. In the present study, more than $95 \%$ of mothers initiated breast-feeding postpartum, which is close to the rate in other reports in China ${ }^{(4,34)}$. Moreover, no statistical difference was observed in the rates of breast-feeding initiation among the women of different pre-pregnancy BMI categories. The high rates of breastfeeding initiation for all pre-pregnancy BMI groups mainly resulted from the Chinese government's support for the Baby-Friendly Hospital Initiative ${ }^{(3)}$, which promotes breastfeeding in hospitals. However, our findings indicate that pre-pregnancy obesity seemed to increase the risk of delayed lactogenesis II, which is consistent with the results of two studies with small sample sizes ${ }^{(23,24)}$.

Table 2 Relative risk (RR) of sustained exclusive breast-feeding at 1, 3 and 6 months postpartum according to pre-pregnancy BMI category in women initiating breast-feeding $(n$ 3065) from the Ma'anshan Birth Cohort Study

\begin{tabular}{|c|c|c|c|c|c|c|c|}
\hline & \multicolumn{7}{|c|}{ Pre-pregnancy BMI category } \\
\hline & \multicolumn{2}{|c|}{ Underweight } & \multirow[b]{2}{*}{ Normal weight† } & \multicolumn{2}{|c|}{ Overweight } & \multicolumn{2}{|c|}{ Obese } \\
\hline & $\mathrm{RR}$ & $95 \% \mathrm{Cl}$ & & $\mathrm{RR}$ & $95 \% \mathrm{Cl}$ & $\mathrm{RR}$ & $95 \% \mathrm{Cl}$ \\
\hline \multicolumn{8}{|c|}{1 month postpartum } \\
\hline Unadjusted & 1.02 & $0.85,1.22$ & 1.00 & 1.08 & $0.84,1.38$ & $1.62^{*}$ & $1.01,2.59$ \\
\hline Adjusted $\ddagger$ & 1.08 & $0.90,1.30$ & 1.00 & 1.01 & $0.78,1.29$ & $1 \cdot 30$ & $0 \cdot 80,2 \cdot 12$ \\
\hline \multicolumn{8}{|c|}{3 months postpartum } \\
\hline Unadjusted & 1.03 & $0.86,1.24$ & 1.00 & $1 \cdot 20$ & $0.94,1.53$ & $1 \cdot 35$ & $0.86,2 \cdot 11$ \\
\hline Adjustedł & 1.07 & $0.88,1.29$ & 1.00 & $1 \cdot 11$ & $0.86,1.43$ & 1.09 & $0.68,1.75$ \\
\hline \multicolumn{8}{|c|}{6 months postpartum } \\
\hline Unadjusted & 0.77 & $0.59,1.01$ & 1.00 & 0.86 & $0.59,1.26$ & 0.73 & $0.38,1.41$ \\
\hline Adjusted $\neq$ & 0.76 & $0.58,1.01$ & 1.00 & 0.85 & $0.57,1.25$ & 0.70 & $0.36,1.37$ \\
\hline
\end{tabular}

${ }^{\star} P<0.05$.

†Reference category.

$\ddagger$ Adjusted for maternal age, gestational age, birth weight, mode of delivery, infant sex, gestational weight gain, ethnicity, education, monthly income of family, parity, cigarette smoking, alcohol drinking, pregnancy-induced hypertension and gestational diabetes. 


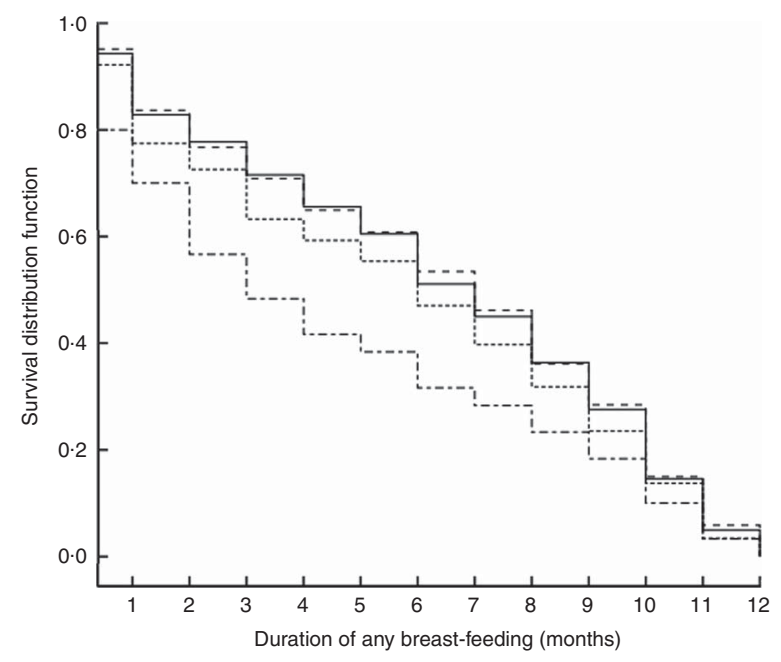

Fig. 3 Kaplan-Meier curves showing the proportion of infants who continued any breast-feeding according to pre-pregnancy BMI category (- - - - - , underweight; _- normal weight; - - - - -, overweight; - . - - , obese) in women initiating breastfeeding $(n$ 3065) from the Ma'anshan Birth Cohort Study

The mean duration of $\mathrm{ABF}$ in the majority of cities or provinces of China is 7-9 months ${ }^{(3)}$. Similarly, the median duration of $\mathrm{ABF}$ in our study was 7 months. We found that obese women were at a higher risk of earlier termination of $\mathrm{ABF}$, compared with normal-weight women, and this finding is consistent with that of a large prospective study in American women ${ }^{(11)}$. Studies evaluating Danish ${ }^{(9)}$, Norwegian $^{(16)}$ and Australian ${ }^{(35)}$ populations have also found that being overweight is related to a shorter duration of ABF. Moreover, previous studies have indicated a negative association between pre-pregnancy BMI and the duration of $\mathrm{EBF}^{(8,33,36,37)}$. However, this observation was not found in the present study. The possible reasons for such inconsistent results include the different study designs, measures of maternal BMI and adjustments for confounding factors. Moreover, ethnic or cultural variation may partly explain the difference. Many studies have shown that pre-pregnancy BMI is negatively associated with breast-feeding outcomes in Caucasian women, but not in African-American women ${ }^{(12-14)}$.

The present study showed that GWG was not associated with the duration of $\mathrm{ABF}$ or $\mathrm{EBF}$, which is supported by most observational studies ${ }^{(9,18,19)}$. Although two studies have indicated that excessive GWG has a negative effect on full breast-feeding ${ }^{(16)}$ and $\mathrm{ABF}^{(16,17)}$, significantly higher risks were found among most GWG categories of overweight or obese women only in comparison with the reference group of normal-weight women who gained the recommended weight. The residual confounding effect of pre-pregnancy BMI was not adequately controlled for. For example, the difference in breast-feeding duration between obese women with excessive GWG and normalweight women with adequate GWG may be attributed to high BMI rather than excessive GWG. Another study showed that women with either insufficient or excessive weight gain had significantly shorter breast-feeding duration than did those who gained the recommended gestational weight ${ }^{(11)}$. Although the breast-feeding duration of the GWG groups differed by less than 1 week, the differences were statistically significant because of the large sample size ( $n$ 13234).

The mechanisms underlying the association of maternal obesity with poor breast-feeding practice are still not clearly understood. Because of the effect of fat mass on prolactin and oxytocin levels, obese women are more likely to have delayed lactogenesis II than are normalweight women ${ }^{(38)}$, which was confirmed in our study. Women with delayed lactogenesis II exhibit shorter breastfeeding duration ${ }^{(20)}$. By contrast, some studies have shown that obese women are less confident of having sufficient milk supply ${ }^{(39)}$ and exhibit a reduced prolactin response to suckling ${ }^{(40)}$, which may contribute to early lactation failure. Large breasts or flat nipples also make it difficult to establish copious milk production ${ }^{(41)}$. Furthermore, pre-pregnancy obese women have a significantly higher risk of adverse health outcomes, including gestational diabetes, pregnancy-induced hypertension, pre-eclampsia and caesarean delivery ${ }^{(42,43)}$, which can confound the relationship between maternal obesity and breast-feeding. In particular, caesarean delivery is being performed ever more frequently in China ${ }^{(44,45)}$. In our study population, obese women had a higher rate of caesarean delivery than did normal-weight women. However, the significance of the association between pre-pregnancy BMI and ABF did not change after this factor was added to the statistical models. This suggested that caesarean delivery, although more prevalent in obese women, did not play a determinant role in the association between pre-pregnancy $\mathrm{BMI}$ and termination of $\mathrm{ABF}$ in our study population.

The major strength of our study is the prospective cohort study design. This design enabled the measurement of the exposure and mediators before the outcome and thus facilitated the assessment of the risk. However, the current study has several limitations that influence the interpretation and generalizability of the results. Because of the low prevalence of pre-pregnancy overweight and obese women in our cohort, the lack of power may have limited our ability to study breast-feeding duration. In addition, the self-reported weight before pregnancy might be underestimated in overweight or obese women ${ }^{(46,47)}$, potentially resulting in non-differential misclassification bias. Despite this limitation, the self-reported prepregnancy weight is widely used in observational studies, because the reporting error is a small percentage of the total body weight ${ }^{(48)}$. The present study did not include psychological factors such as body perception and social support of breast-feeding, which may affect the success of breast-feeding. Although the study also did not include other pregnancy-related psychological factors such as 
depressive symptoms, perceived stress and anxiety, most reports have indicated that these factors do not mediate the relationship between pre-pregnancy BMI and breastfeeding ${ }^{(31,37)}$. Finally, our study was limited to the use of geographic origin as a means of exploring Chinese women in our sample.

\section{Conclusion}

The present study showed that pre-pregnancy obesity, but not GWG, exerted a negative effect on breast-feeding in Chinese women. Pre-pregnancy obesity increased the risks of delayed lactogenesis II and early termination of $\mathrm{ABF}$. The clear implication of our findings is that a healthy weight should be maintained for women before conception. Because of the increasing obesity rate in women, at-risk individuals should be identified for more targeted clinic interventions to support breast-feeding practices.

\section{Acknowledgements}

Acknowledgements: The authors acknowledge and extend their appreciation to all of the participants in the Ma'anshan Birth Cohort Study. Financial support: This research was funded by the National Natural Science Foundation of China (grant number NSCF-81573168). The National Natural Science Foundation of China had no role in the design, analysis or writing of this article. Conflict of interest: The authors declare that they have no conflicts of interest. Authorship: F.-B.T., X.-Y.T. and K.H. designed the study. F.-B.T., X.-Y.T., K.H., S.-Q.Y., A.-Z.Z., R.-W.T., H.C. and C.-L.G. generated, collected, analysed and/or interpreted the data. F.-B.T., X.-Y.T., A.-Z.Z. and R.-W.T. drafted or revised the initial manuscript. All authors read and approved the final manuscript. Ethics of human subject participation: All protocols, instruments and the process for obtaining informed consent for this study were approved by the Biomedical Ethics Committee of Anhui Medical University in China.

\section{References}

1. Centers for Disease Control and Prevention (2015) Breastfeeding Among US Children Born 2002-2012, CDC National Immunization Survey. http://www.cdc.gov/breastfeeding/ data/NIS_data/index.htm (accessed June 2016).

2. Bagci Bosi AT, Eriksen KG, Sobko T et al. (2016) Breastfeeding practices and policies in WHO European Region Member States. Public Health Nutr 19, 753-764.

3. Xu F, Qiu L, Binns CW et al. (2009) Breastfeeding in China: a review. Int Breastfeed J 4, 6.

4. Guo S, Fu X, Scherpbier RW et al. (2013) Breastfeeding rates in central and western China in 2010: implications for child and population health. Bull World Health Organ 91, 322-331.

5. Liu Y, Dai W, Dai X et al. (2012) Prepregnancy body mass index and gestational weight gain with the outcome of pregnancy: a 13-year study of 292,568 cases in China. Arch Gynecol Obstet 286, 905-911.

6. Xi B, Liang Y, He $\mathrm{T}$ et al. (2012) Secular trends in the prevalence of general and abdominal obesity among Chinese adults, 1993-2009. Obes Rev 13, 287-296.

7. Turcksin R, Bel S, Galjaard S et al. (2014) Maternal obesity and breastfeeding intention, initiation, intensity and duration: a systematic review. Matern Child Nutr 10, 166-183.

8. Hauff LE, Leonard SA \& Rasmussen KM (2014) Associations of maternal obesity and psychosocial factors with breastfeeding intention, initiation, and duration. Am J Clin Nutr 99, 524-534.

9. Baker JL, Michaelsen KF, Sorensen TI et al. (2007) High prepregnant body mass index is associated with early termination of full and any breastfeeding in Danish women. Am J Clin Nutr 86, 404-411.

10. Newby RM \& Davies PS (2016) Antenatal breastfeeding intention, confidence and comfort in obese and non-obese primiparous Australian women: associations with breastfeeding duration. Eur J Clin Nutr 70, 935-940.

11. Li R, Jewell S \& Grummer-Strawn L (2003) Maternal obesity and breast-feeding practices. Am J Clin Nutr 77, 931-936.

12. Masho SW, Cha S \& Morris MR (2015) Prepregnancy obesity and breastfeeding noninitiation in the United States: an examination of racial and ethnic differences. Breastfeed Med 10, 253-262.

13. Kugyelka JG, Rasmussen KM \& Frongillo EA (2004) Maternal obesity is negatively associated with breastfeeding success among Hispanic but not Black women. J Nutr 134, 1746-1753.

14. Liu J, Smith MG, Dobre MA et al. (2010) Maternal obesity and breast-feeding practices among white and black women. Obesity (Silver Spring) 18, 175-182.

15. Wojcicki JM (2011) Maternal prepregnancy body mass index and initiation and duration of breastfeeding: a review of the literature. J Womens Health (Larchmt) 20, 341-347.

16. Winkvist A, Brantsaeter AL, Brandhagen $\mathrm{M}$ et al. (2015) Maternal prepregnant body mass index and gestational weight gain are associated with initiation and duration of breastfeeding among Norwegian mothers. I Nutr 145, 1263-1270.

17. Hilson JA, Rasmussen KM \& Kjolhede CL (2006) Excessive weight gain during pregnancy is associated with earlier termination of breast-feeding among White women. $J$ Nutr 136, 140-146.

18. Castillo H, Santos IS \& Matijasevich A (2016) Maternal prepregnancy BMI, gestational weight gain and breastfeeding. Eur J Clin Nutr 70, 431-436.

19. Manios Y, Grammatikaki E, Kondaki K et al. (2009) The effect of maternal obesity on initiation and duration of breast-feeding in Greece: the GENESIS study. Public Health Nutr 12, 517-524.

20. Brownell E, Howard CR, Lawrence RA et al. (2012) Delayed onset lactogenesis II predicts the cessation of any or exclusive breastfeeding. J Pediatr 161, 608-614.

21. Dewey KG, Nommsen-Rivers LA, Heinig MJ et al. (2003) Risk factors for suboptimal infant breastfeeding behavior, delayed onset of lactation, and excess neonatal weight loss. Pediatrics 112, 607-619.

22. Nommsen-Rivers LA, Chantry CJ, Peerson JM et al. (2010) Delayed onset of lactogenesis among first-time mothers is related to maternal obesity and factors associated with ineffective breastfeeding. Am J Clin Nutr 92, 574-584.

23. Hilson JA, Rasmussen KM \& Kjolhede CL (2004) High prepregnant body mass index is associated with poor lactation outcomes among white, rural women independent of psychosocial and demographic correlates. J Hum Lact 20, 18-29.

24. Chapman DJ \& Perez-Escamilla R (1999) Identification of risk factors for delayed onset of lactation. J Am Diet Assoc 99, 450-454. 
25. Matias SL, Dewey KG, Quesenberry CP Jr et al. (2014) Maternal prepregnancy obesity and insulin treatment during pregnancy are independently associated with delayed lactogenesis in women with recent gestational diabetes mellitus. Am J Clin Nutr 99, 115-121.

26. Yang S, Peng A, Wei S et al. (2015) Pre-pregnancy body mass index, gestational weight gain, and birth weight: a cohort study in China. PLoS One 10, e0130101.

27. Chapman DJ \& Perez-Escamilla R (2000) Maternal perception of the onset of lactation is a valid, public health indicator of lactogenesis stage II. J Nutr 130, 2972-2980.

28. Perez-Escamilla R \& Chapman DJ (2001) Validity and public health implications of maternal perception of the onset of lactation: an international analytical overview. J Nutr 131, issue $11,3021 \mathrm{~S}-3024 \mathrm{~S}$.

29. World Health Organization (2008) Indicators for assessing infant and young child feeding practices. Part 1: Definitions. http://whqlibdoc.who.int/publications/2008/9789241596664_ eng.pdf (accessed June 2016).

30. Zhu P, Hao J, Jiang X et al. (2013) New insight into onset of lactation: mediating the negative effect of multiple perinatal biopsychosocial stress on breastfeeding duration. Breastfeed Med 8, 151-158.

31. Mehta UJ, Siega-Riz AM, Herring AH et al. (2011) Maternal obesity, psychological factors, and breastfeeding initiation. Breastfeed Med 6, 369-376.

32. Thompson LA, Zhang S, Black E et al. (2013) The association of maternal pre-pregnancy body mass index with breastfeeding initiation. Matern Child Health J 17, 1842-1851.

33. Guelinckx I, Devlieger R, Bogaerts A et al. (2012) The effect of pre-pregnancy BMI on intention, initiation and duration of breast-feeding. Public Health Nutr 15, 840-848.

34. Liu P, Qiao L, Xu F et al. (2013) Factors associated with breastfeeding duration: a 30-month cohort study in northwest China. J Hum Lact 29, 253-259.

35. Oddy WH, Li J, Landsborough L et al. (2006) The association of maternal overweight and obesity with breastfeeding duration. J Pediatr 149, 185-191.

36. Martinez JL, Chapman DJ \& Perez-Escamilla R (2016) Prepregnancy obesity class is a risk factor for failure to exclusively breastfeed at hospital discharge among Latinas. J Hum Lact 32, 258-268.
37. Mehta UJ, Siega-Riz AM, Herring AH et al. (2012) Pregravid body mass index, psychological factors during pregnancy and breastfeeding duration: is there a link? Matern Child Nutr 8, 423-433.

38. Amir LH \& Donath S (2007) A systematic review of maternal obesity and breastfeeding intention, initiation and duration. BMC Pregnancy Childbirth 7,9.

39. Mok E, Multon C, Piguel L et al. (2008) Decreased full breastfeeding, altered practices, perceptions, and infant weight change of prepregnant obese women: a need for extra support. Pediatrics 121, e1319-e1324.

40. Rasmussen KM \& Kjolhede CL (2004) Prepregnant overweight and obesity diminish the prolactin response to suckling in the first week postpartum. Pediatrics 113, e465-e471.

41. Jevitt C, Hernandez I \& Groer M (2007) Lactation complicated by overweight and obesity: supporting the mother and newborn. J Midwifery Womens Health 52, 606-613.

42. Rahman MM, Abe SK, Kanda M et al. (2015) Maternal body mass index and risk of birth and maternal health outcomes in low- and middle-income countries: a systematic review and meta-analysis. Obes Rev 16, 758-770.

43. Yu Z, Han S, Zhu J et al. (2013) Pre-pregnancy body mass index in relation to infant birth weight and offspring overweight/obesity: a systematic review and meta-analysis. PLOS One 8, e61627.

44. Feng XL, Xu L, Guo Y et al. (2012) Factors influencing rising caesarean section rates in China between 1988 and 2008. Bull World Health Organ 90, 30-39, 39A.

45. Feng XL, Wang Y, An L et al. (2014) Cesarean section in the People's Republic of China: current perspectives. Int $J$ Womens Health 6, 59-74.

46. Hill A \& Roberts J (1998) Body mass index: a comparison between self-reported and measured height and weight. J Public Health Med 20, 206-210.

47. Paxton SJ, Sculthorpe A \& Gibbons K (1994) Weight-loss strategies and beliefs in high and low socioeconomic areas of Melbourne. Aust J Public Health 18, 412-417.

48. Harris HE \& Ellison GT (1998) Practical approaches for estimating prepregnant body weight. J Nurse Midwifery $\mathbf{4 3}$, 97-101. 